\title{
Topic Modeling of Chinese Language Beyond a Bag-of-Words
}

\author{
Zengchang Qin ${ }^{\mathrm{a}, *}$, Yonghui Cong ${ }^{\mathrm{a}}$, Tao Wan ${ }^{\mathrm{b}}$ \\ ${ }^{a}$ Intelligent Computing and Machine Learning Lab, School of Automation Science and \\ Electrical Engineering, Beihang University, Beijing, China. \\ ${ }^{b}$ School of Biological Science and Medical Engineering, Beihang University, Beijing, China.
}

\begin{abstract}
The topic model is one of best known hierarchical Bayesian models for language modeling and document analysis. It has achieved a great success in text classification, in which a text is represented as a big of its words, disregarding grammar and even word order, that is referred to as the bag-of-words assumption. In this paper, we investigate topic modeling of the Chinese language, which has different morphology from alphabetical western languages like English. The Chinese characters, but not the Chinese words, are the basic structural units in Chinese. In previous empirical studies, it shows that the character-based topic model performs better than the word-based topic model. In this research, we propose the character-word topic model (CWTM) to consider the character-word relation in topic modeling. Two types of experiments are designed to test the performance of the new proposed model: topic extraction and text classification. By empirical studies, we demonstrate the superiority of the new proposed model comparing to both word and character based topic models.
\end{abstract}

Keywords: Topic models, Chinese language modeling, Text classification, Language model, Character-word topic model, Latent Dirichlet allocation

\footnotetext{
* Corresponding author

Email addresses: zcqin@buaa.edu.cn (Zengchang Qin), yonghuicong@gmail.com (Yonghui Cong), tao.wan.wan@gmail.com (Tao Wan)
} 


\section{Introduction}

Topic models (Blei and Lafferty, 2009; Steyvers and Griffiths, 2007) are a class of hierarchical probabilistic models for analyzing discrete data collections. They have attracted lots of attention in recent years for modeling documents in semantic level. The main idea of topic model is that a document can be represented as a mixture of some latent topics, and each topic is a probability distribution over the vocabulary. Latent Dirichlet Allocation (LDA) (Blei et al., 2003), which is a typical and wide-used topic model, depicts a probabilistic procedure that how documents are generated.

LDA can be regarded as an extension of Probabilistic Latent Semantic Analysis (PLSA) (Thomas, 2001). By virtue of the Dirichlet priors that are placed on both the document-topic relation and the topic-word relation, LDA is perceived as fully generative topic model by defining a complete generative process with full Bayesian estimators. Especially, the Dirichlet prior of document-topic distribution free the model from specific training data. There are numerous research by infusing the model with additional information, such as syntax information for part-of-speech tagging (Darling and Song, 2013) and text tags recommendation in Twitters. Specifically, (Ding et al., 2012) realize tags recommendation in Sina Weibo ${ }^{1}$ It is worth emphasizing that topic models based on parallel computing began to get more attention under the demand of big data processing. Parallel LDA (PLDA) (Wang et al., 2013) is a parallel topic model based on the MapReduce framework. It shows good applicability for the modeling and analysis of massive text processing in the era of big data.

In natural language processing, an English text can be regarded as a collection of words, which are the basic semantic units in the majority of western languages. However, the morphology of Chinese language is more complex. A Chinese syllable corresponds to a Chinese character, some characters stand alone as an individual word. A Chinese word can consist of more than one

\footnotetext{
${ }^{1}$ Sina Weibo is a Twitter-like social network service in China.
} 
characters, usually, two, but there can be three or more ${ }^{2}$. A Chinese document need to be segmented into words as there is no spaces between words in a Chinese text. The Chinese words generally have more specific meanings than the Chinese characters. Most previous research applying topic models to analyze Chinese documents choose Chinese words as the basic terms. However, word-based methods completely ignore the fact that a Chinese word is composed of Chinese characters. Words sharing the same character may have some semantic relations, such a relation cannot be detected in word-based models. For example, the Chinese words "da xue" (university) and "xue yuan" (college) are literally related by sharing the same character "xue", which means study or learn, and they are also semantically related in meaning and may have a high probability to occur in the same context. However, in word-based computational models, these two words are treated as two distinct words that has no relations at all. Some recent research shows that, the Chinese characters, not words, are the basic structure units for the Chinese language. This has been both discussed in Chinese linguistics (Xu, 2001) and testified using computational model (Zhao et al., 2011a,b).

With the constantly increasing of new Chinese words, the size of word vocabulary can be larger and larger while the number of commonly used Chinese characters is still the same around $3000^{3}$. It is attributed to the fact that characters constituting new words probably have already appeared in the words. In the standard LDA (Blei et al., 2003) model, all those words unseen in training data are assigned the equal probability in a topic by simply placing a symmetric Dirichlet prior (Minka, 2000) on the topic-word distribution. Regardless of their component characters having different occurrence rates in the training data. Therefore, taking Chinese character as the basic unit of topic models

\footnotetext{
${ }^{2}$ For example, most Chinese idioms are consist of 4 Chinese characters.

${ }^{3}$ Previous research has reported that 3500 most frequently used characters can cover $99.48 \%$ of a written materials corpus over 2 million characters and 1000 most used characters can cover $90 \%$ of a corpus of daily life with over a billion characters Wikipedia (2015).
} 
could be an approach to tackle the above problem (Xu, 2001; Zhang and Qin, 2010). Chinese characters contain important semantic meaning. However, its limitation is obvious: a considerable number of Chinese words have the semantic meaning irrelevant to the meanings of its component characters. For instance, "ke le", which is a transliteration of Coke Cola, its semantic meaning has no relation with both "ke" (may, allow) and "le" (happy). The reason for using these two characters is because its pronounce similarly to "cola" and literally means happy in Chinese. For such cases, embedding word-character relations will bring noise and may not yield better results.

Chinese characters-based NLP research is not a very developed field. As the classical NLP research is based on alphabetical languages (mainly English), though Chinese morphology is quite different, most NLP studies in Chinese focus on using Chinese words by segmenting sentences, rare studies have been done to focus on the characters. A book written by Wong (Wong et al., 2009) focuses on Chinese NLP and the unique properties of Chinese language which offers careful comparisons with English wherever necessary. It contains a concise and insightful linguistic analysis of the problems faced by Chinese NLP.

In this paper, we first conduct a series of experiments of topic modeling based on Chinese characters and words, respectively. The performance of the character-based and the word-based topic models in text classification are compared. Based on classical topic model, we propose the character-word topic model (CWTM) by considering the character-word relation in topic modeling. Two types of experiments are designed to test the performance of the new proposed model: topic extraction and text classification. The results on both tests demonstrate the effectiveness of the new proposed model.

The remainder of this paper is organized as follows: In Section 2, we will introduce the standard LDA model and discuss the differences between wordbased and character-based LDA models in details. The relationship between Chinese characters and words is considered to develop a new topic model in Section 3. In Section 4, we apply Gibbs Sampling to inference the new model. We then conduct two types of experiments and the results are analyzed and 
discussed in Section 5. The conclusions and future work are given in the end.

\section{Generative Models of Topics}

Topic model is based on the Bag-of-Words (BoW) assumption. The essential idea of topic model is that a document can be represented by a mixture of latent topics and each topic is a distribution over words. Probabilistic Latent Semantic Analysis (PLSA) (Thomas, 2001) is attached much attention in early research which is modified from classical Latent Semantic Analysis (LSA) (Deerwester et al., 1990) by introducing a latent topic variable. Along with the Latent Dirichlet Allocation (LDA) (Blei et al., 2003) is put forward, the problem of the linearly growth of parameters number in PLSA is solved.

\subsection{Latent Dirichlet Allocation}

LDA is a generative topic model that treats the topic mixture weights for a document as a $D$-dimensional random variable $\theta$ and weights over words for each topic as a $K$-dimensional random variable $\varphi$ with Dirichlet prior distributions, respectively. Where $D$ is the document number and $K$ is the topic number.

Dirichlet distribution is a family of continuous multivariate exponential probability distribution over the simplex of positive vectors that sums to one. Symmetric Dirichlet prior is assumed with concentration parameter in almost all previous work on LDA. Take $\theta=\left\{\theta_{1}, \ldots, \theta_{D}\right\}$ for example,

$$
p(\theta \mid \alpha)=\operatorname{Dir}(\alpha)=\frac{\Gamma\left(\sum_{d=1}^{D} \alpha_{d}\right)}{\prod_{d=1}^{D} \Gamma\left(\alpha_{d}\right)} \theta_{1}^{\alpha_{1}-1} \ldots \theta_{D}^{\alpha_{D}-1}
$$

Where $\theta_{d} \geq 0, \sum_{d=1}^{D} \theta_{d}=1, \alpha_{d}>0$ and $\Gamma(x)$ is the Gamma function. The prior over $\varphi=\left\{\varphi_{1}, \ldots, \varphi_{K}\right\}$ is also assumed to be a symmetric Dirichlet with parameter $\beta$ as similar with $\theta$.

The tokens in document $d$ : $\mathbf{W}^{(d)}=\left\{W_{i}^{(d)}\right\}_{i=1}^{N_{d}}$ are associated with corresponding topic assignments $\mathbf{Z}^{(d)}=\left\{Z_{i}^{(d)}\right\}_{i=1}^{N_{d}}$ drawn from the document-specific distribution over topics. $N_{d}$ is the word number of document $d$. While the 


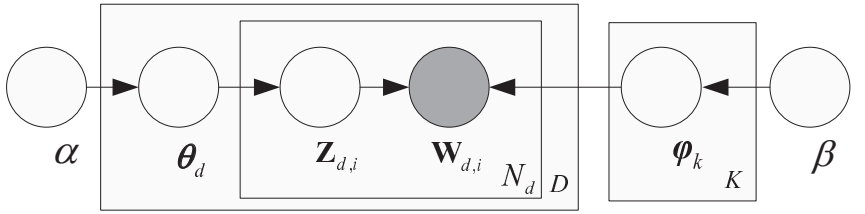

Figure 1: Graphical representation of the standard LDA (Blei et al., 2003).

tokens are drawn from the topics distribution over words $\varphi=\left\{\varphi_{1}, \ldots, \varphi_{K}\right\}$ :

$$
\begin{aligned}
p\left(\mathbf{Z}^{(d)} \mid \theta_{d}\right) & =\prod_{i=1}^{N_{d}} \theta_{Z_{i}^{(d)}} \\
p\left(\mathbf{W}^{(d)} \mid \mathbf{Z}^{(d)}, \varphi\right) & =\prod_{i=1}^{N_{d}} \varphi_{W_{i}^{(d)} \mid Z_{i}^{(d)}}
\end{aligned}
$$

Fig. 1 illustrates this process using a graphical model. And the generative process for a corpus is showed in the Algorithm 1. Based on this procedure, we can calculate the joint probability of $\theta, \mathbf{Z}^{(d)}$ and $\mathbf{W}^{(d)}$ given $\alpha$ and $\beta$ as hyper-parameters as follows:

$$
p\left(\theta_{d}, \mathbf{Z}^{(d)}, \mathbf{W}^{(d)} \mid \alpha, \beta\right)=p\left(\theta_{d} \mid \alpha\right) \prod_{i=1}^{N_{d}} p\left(Z_{i}^{(d)} \mid \theta_{d}\right) p\left(w_{i}^{(d)} \mid Z_{i}^{(d)}, \beta\right)
$$

The marginal distribution of $\mathbf{W}^{(d)}$ can be calculated by summing over $Z_{i}^{(d)}$ and integrating over $\theta_{d}$ by the following equation:

$$
p(\mathbf{W} \mid \alpha, \beta)=\int p(\theta \mid \alpha) \prod_{i=1}^{N_{d}} \sum_{Z_{i}} p\left(Z_{i} \mid \theta\right) p\left(W_{i} \mid Z_{i}, \beta\right) d \theta
$$

In real text corpus, documents and tokens are observed while the corresponding topics $\mathbf{Z}$ are unobserved. Some effective inference learning methods such as Variable Inference (Blei et al., 2003; Teh et al., 2006b), Markov Chain Monte Carlo (MCMC) (Griffiths and Steyvers, 2004) - especially Gibbs sampling (Cowans, 2006; Teh et al., 2006a; Geman and Geman, 1984) - and Expectation Propagation (EP) (Minka and Lafferty, 2002) are utilized to infer the latent topics. 


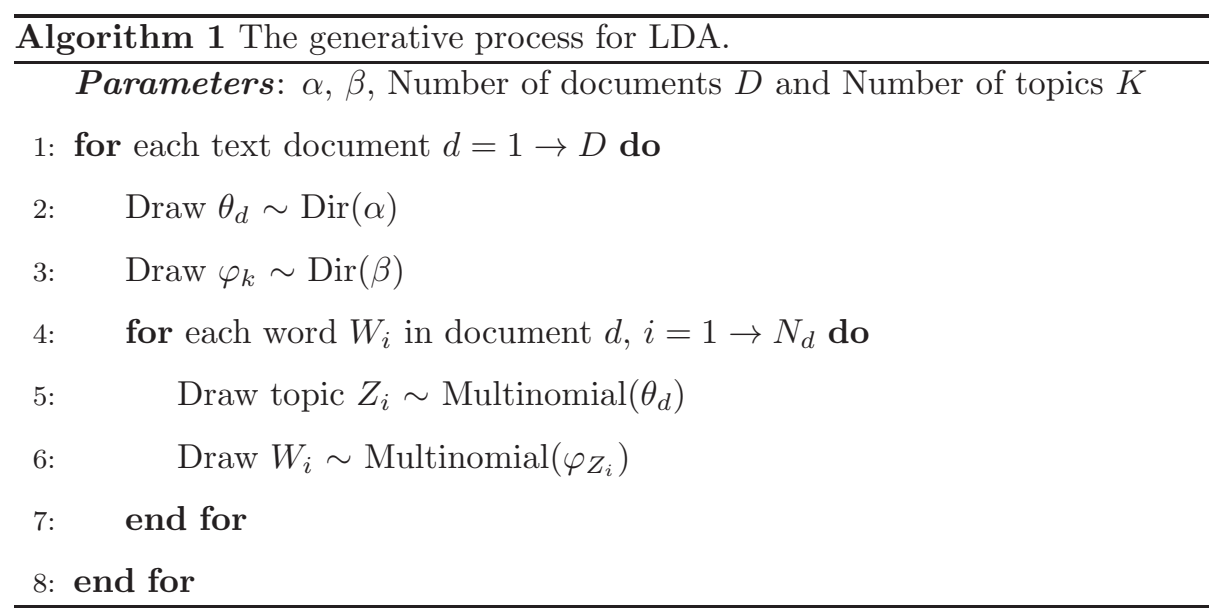

\subsection{Word-based and Character-based LDA}

In text modeling, a document is fairly reasonable treated as a bag-of-words for English corpus or other alphabetical languages. However, the morphology of Chinese language is different as we have explained in Section 1. Many Chinese words are combination of characters while the meanings are sort of related to these combinations. Different matching of characters leads different meaning for the same character. In modern Chinese language, a character has a broad range of sense that is open to be combined with other characters to be more semantically specific so as to some new words are introduced to vocabulary. For example, "lei ren", "gei li" are new words appeared from the Internet in recent years. But all the component characters are commonly used words for ages. Actually almost all Chinese words are made of grouping and ordering of two or more Chinese characters which are heavily depending on each other.

As we have seen from above explanation, a Chinese word can be considered as a compound of characters in different ways. Fig. 2 demonstrate examples of compound words of using "bao". When matching with the character "qian" (money), "shu" (book) and "pi" (leather), it all means "bag". When matching with "zhuang" (assemble), "wei" (enclose) and "za" (tie) it means "wrap" (or make it a bag). Each Chinese character carries ambiguous semantic meaning, by forming a word, the semantic meaning is refined. We will apply Chinese words 
Figure 2: An example of compound word of using "bao" as the center character.

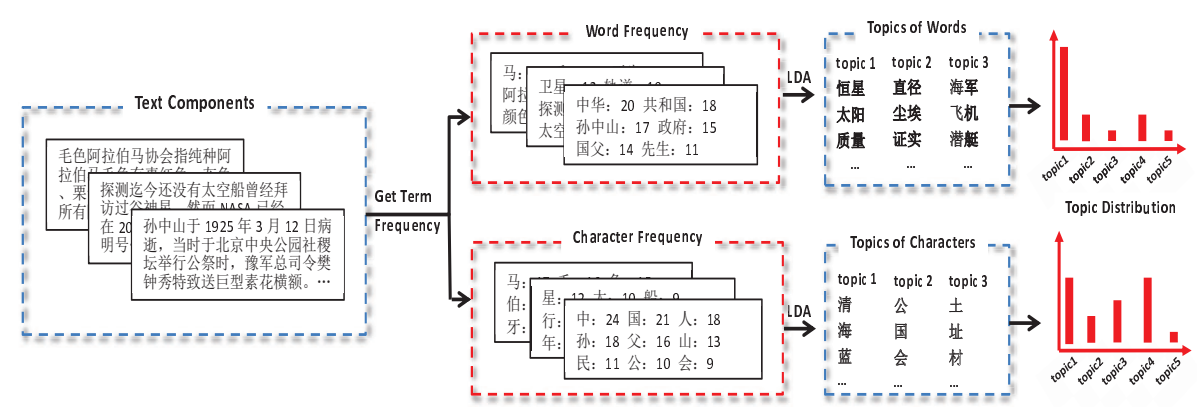

Figure 3: Chinese representation based on Latent Dirichlet Allocation with words and characters respectively. In word-based LDA, Chinese words are treated as the basic unit by segmentation and topic-word distribution is obtained. While in the character-based LDA, a text is regarded as a collection of characters, and the final result is topic-character distribution.

and characters to the LDA model, respectively, to compare their performance. The process of modeling for the text components based on Chinese words and characters is schematically showed in Fig. 3. It shows the differences between topics of modeling Chinese texts segmented with words and with characters.

\section{Character-Word Topic Model}

As we have discussed in the above sections, neither characters nor words based models consider the character-word relations and the latent semantic relationships were ignored in previous works. In this section, we are going to develop a new topic model by modeling such character-word relations in the Chinese language. 


\begin{tabular}{cc|cc||cc|cc}
\hline \multicolumn{4}{c}{ NEWS1W } \\
\hline Character & 4142 & Word & 25673 & Character & 4825 & Word & 48506 \\
\hline 一 & 38 & 一一 & 5147 & 一 & 183 & 一一 & 5367 \\
丁 & 1586 & 一万 & 5376 & 丁 & 1041 & 一万 & 5020 \\
七 & 782 & 一个 & 23 & 七 & 455 & 一个 & 185 \\
万 & 355 & 丁香 & 20545 & 万 & 833 & 丁二酸 & 19123 \\
丈 & 1811 & 七十 & 11047 & 丈 & 781 & 七七事变 & 24394 \\
三 & 288 & 万分 & 14332 & 三 & 3 & 万亿 & 23458 \\
上 & 40 & 三十 & 702 & 上 & 76 & 三十 & 11401 \\
下 & 42 & 上乘 & 14972 & 下 & 380 & 上乘 & 35623 \\
不 & 232 & 下发 & 7613 & 不 & 105 & 下发 & 35119 \\
与 & 944 & 不利 & 4441 & 与 & 1132 & 不利 & 1674 \\
\hline
\end{tabular}

Figure 4: Learned dictionaries of Chinese word and character dictionaries. The items in dictionary are ordered associated with their occurrences. orders.

\subsection{Character-Word Relation}

What we want to see is when the probability of a word is high in a topic, so are its component characters in the same topic by considering the characterword relations and the latent semantic relationships. We utilize statistical count method to identify the relation representation as $R . R=\left\{R_{i j}, i=1,2, \ldots, V_{w}\right.$, $\left.j=1,2, \ldots, V_{c}\right\}$ is a 2 -dimensional matrix containing Chinese character-word composition relationship trained from the dictionary. It is defined as the count of character $C_{j}$ contained in word $W_{i}$ :

$$
R_{i j}=\operatorname{Count}\left(W_{i}, C_{j}\right), i=1,2, \ldots, V_{w}, j=1,2, \ldots, V_{c}
$$

where $V_{w}$ and $V_{c}$ represent the size of Chinese word vocabulary and character vocabulary. The dictionaries are obtained by word or character frequency of training corpus. Some details of Chinese word and character dictionaries of the corpuses, NEWS1W and Ch-Wikipedia, are shown in Fig. 4, in which the items in dictionary are ordered according to their frequency of occurrences.

We assume all characters in a word share the identical topic, which is the same as the word's topic assignment. That is, as showed in Fig. 5, if Chinese 


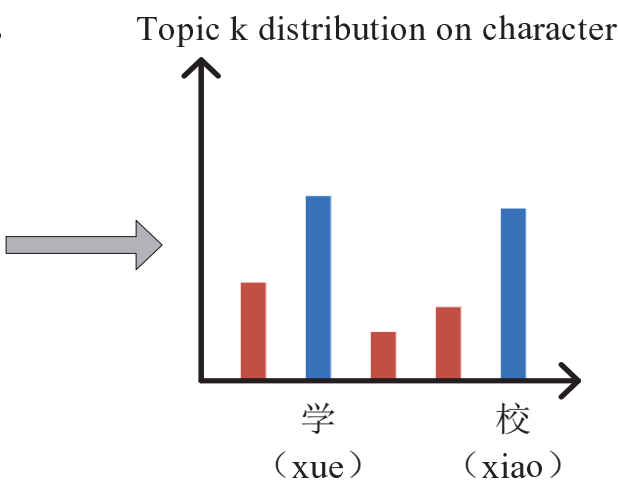

Figure 5: The left-hand side figure shows the $k$ th topic distribution on words and the righthand is on characters. The word and its component characters are assigned to the same topic $k$. Moreover, the larger the probability of the word in the topic, the larger the probability of the component characters.

word "xue xiao" is assigned with topic $k$, then its component characters "xue" and "xiao" are both assigned with topic $k$ as well. We will give the deterministic quantitative definition among topic-character distribution, topic-word distribution and character-word relation in the following part. Now we can improve the standard LDA after building the character-word relation. Compared with the standard LDA in Fig. 1, we make topic-word distribution $\varphi_{W_{k}}$ which infuse topic-character distribution $\varphi_{C_{k}}$ and character-word relation $\mathbf{R}$ replace the $\varphi_{k}$ distribution as is showed in Fig. 6(a).

\subsection{Priors for $C W T M$}

Dirichlet priors are over both $\theta$ and $\varphi$ with fixed concentration parameters $\alpha$ and $\beta$ (Asuncion et al., 2009). Alternatively, the uniform base measures in the Dirichlet priors over $\theta$ and $\varphi$ can be replaced with non-uniform base measures $m$ and $n$, respectively (Wallach et al., 2009). In (Blei and Lafferty, 2006), the document-topic distribution $\theta$ is replaced by natural parameter $\eta$ with $K$ dimensions. $\eta$ can take any value in $K$-dimensional space rather than limited by the probability distribution. Thus in CWTM showed in Fig. 6(a), the uniform base measure in Dirichlet priors over $\theta_{d}$ and $\varphi_{C_{k}}$ also can be replaced with 
non-uniform base measure, separately. An asymmetric prior can be placing over $\varphi_{W_{k}}$ as well. So what are the asymmetric priors good for?

There exist some topics that present in almost all documents (topics which deal with general themes in the collection and topics consisted of stopwords) while most topics are special for documents. As a symmetric prior try to rearrange words by breaking up the very frequent topics and adding some of their words to smaller topics to improve symmetry, it cannot account for that proprietary of topic distributions, which leads to worse or less concise small topics, worse log-likelihood, and apparently worse classification performance for documents. While asymmetric prior means more natural looking of LDA topics. What we expect is certain groups of words - regarded as topics - to be occurring more frequently than others in one document. The CWTM model can now account for that property by learning a non-uniform Dirichlet prior. Therefore, asymmetric priors can generate stable topic distributions and better results under the increment of topic number. It serves to share commonalities across document.

However, it is reasonable that the topic distribution over words doesn't have large variations corpus-wide. We expect a relatively stable topic distribution in words. An asymmetric prior over $\varphi$ is therefore may not be a good idea (Wallach et al., 2009). The corpus-wide word usage statistics will be realized by the base measure and a prior statement about whether topics will have more sparse or more uniform distributions over words is determined by the concentration parameter $\beta$. So the topics are free to be as distinct and specialized as is necessary (Wallach et al., 2009). A symmetric prior serve to avoid conflicts between topics.

Reported in (Wallach et al., 2009), an asymmetric Dirichlet prior over documenttopic distribution has substantial advantages over a symmetric prior, while an asymmetric prior over the topic-word distribution provides no real benefit. To investigate the effects of the priors over $\theta_{d}$ and $\varphi_{W_{k}}$, the four combinations of symmetric and asymmetric Dirichlet priors is listed in Table 1: model with symmetric priors over both $\theta$ and $\varphi_{W_{k}}$ is denoted SS-CWTM, where $S$ means 


\begin{tabular}{c|c|c}
\hline & symmetric priors for $\varphi_{W_{k}}$ & asymmetric priors for $\varphi_{W_{k}}$ \\
\hline symmetric priors for $\theta$ & SS-CWTM & SA-CWTM \\
\hline asymmetric priors for $\theta$ & AS-CWTM & AA-CWTM \\
\hline
\end{tabular}

symmetric. Model with a symmetric prior over $\theta$ and an asymmetric prior over $\varphi_{W_{k}}$ is denoted SA-CWTM, where $A$ means asymmetric. Model with an asymmetric prior over $\theta$ and a symmetric prior over $\varphi_{W_{k}}$ is denoted by AS-CWTM and an asymmetric prior over both $\theta$ and $\varphi_{W_{k}}$ is denoted by AA-CWTM. We compare the structures of the four extended CWTM models in Fig. 6. Dirichlet distribution is denoted by $\operatorname{Dir}(\mathbf{m}, s)$ where $\mathbf{m}$ is the normalized mean $\sum_{i} m_{i}=1$ and $s$ means a scalar parameter called precision. $\mathbf{m} * s$ equals to $\alpha$ in standard LDA (Minka, 2000).

We treat $\mathbf{m}_{\theta}$ and $\mathbf{m}_{W_{k}}$ as unknown and follow Dirichlet priors. The $\mathbf{m}_{W_{k}}$ is obtained according to the character-word relation and topic-character distribution. It is defined by a deterministic function $F$ :

$$
\begin{aligned}
\mathbf{m}_{W_{k, i}} & =F\left(\varphi_{C_{k}}, \mathbf{R}\right)_{i} \\
& =N_{k}\left(\prod_{j=1}^{V_{c}} \varphi_{C_{k, j}} R_{i j}\right)^{\frac{1}{\sum_{j=1}^{V_{c}} R_{i j}}}, \quad i=1,2, \ldots, V_{w}
\end{aligned}
$$

where $N_{k}$ is a constant to ensure all the coordinates of $\mathbf{m}_{W_{k}}$ sums up to $1 . V_{c}$ and $V_{w}$ represent the size of Chinese character vocabulary and word vocabulary. And the character-word relation can be used in other forms by simply altering the definition of function $F$. As testified in Section 5, the SA-CWTM showed in Fig 6(b) has outstanding classification performance, which we prefer to treat as the best CWTM model to compare to.

The generative procedure of this model for a corpus is outlined in Algorithm 2. The major difference between this model and LDA is the prior of the topic-word distribution $\varphi_{W}$ and the infusing of the character-word relation. In LDA, all the $K$ distributions $\varphi_{W_{1}}, \varphi_{W_{2}}, \ldots, \varphi_{W_{K}}$ own a common 


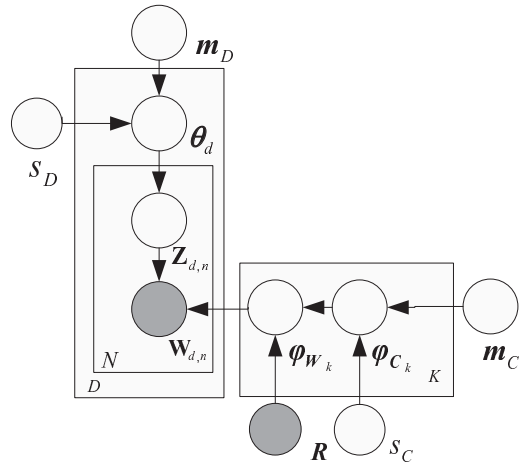

(a) SS-CWTM.

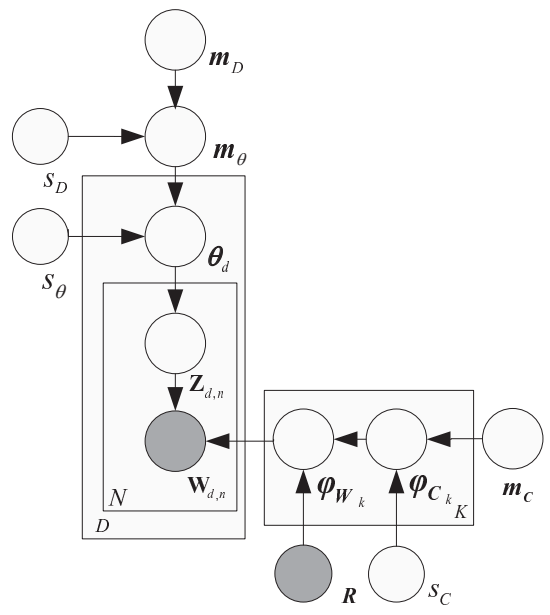

(c) AS-CWTM.

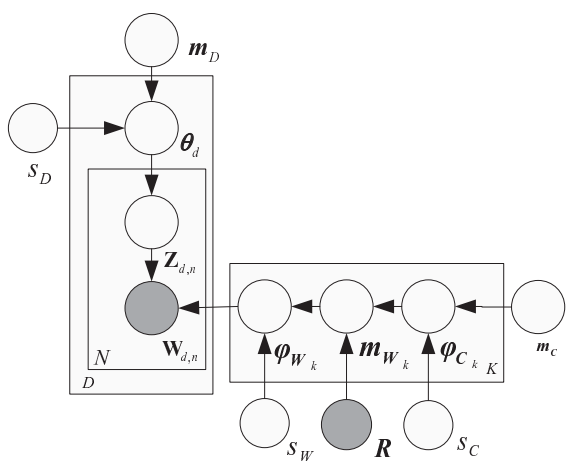

(b) SA-CWTM.

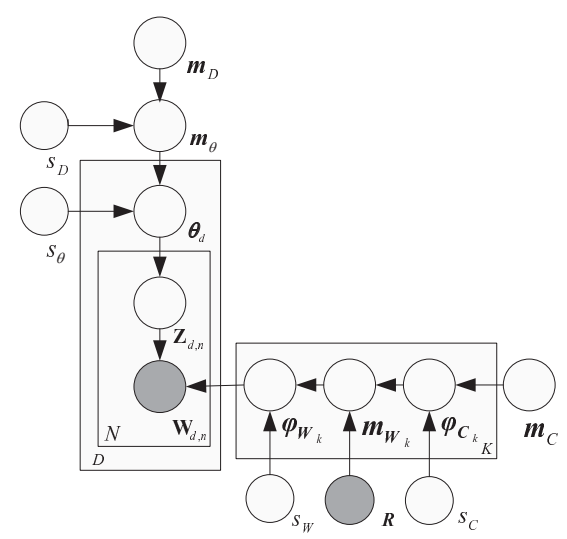

(d) AA-CWTM.

Figure 6: Graphical representation of four variants of the CWTM model: SS-CWTM, SACWTM, AS-CWTM and AA-CWTM. (a) SS-CWTM has symmetric priors over both $\theta$ and $\varphi_{W_{k}}$. (b) SA-CWTM has a symmetric prior over $\theta$ and an asymmetric prior over $\varphi_{W_{k}}$. (c) AS-CWTM has an asymmetric prior over $\theta$ and a symmetric prior over $\varphi_{W_{k}}$. (d) AA-CWTM an asymmetric prior over both $\theta$ and $\varphi_{W_{k}}$. 


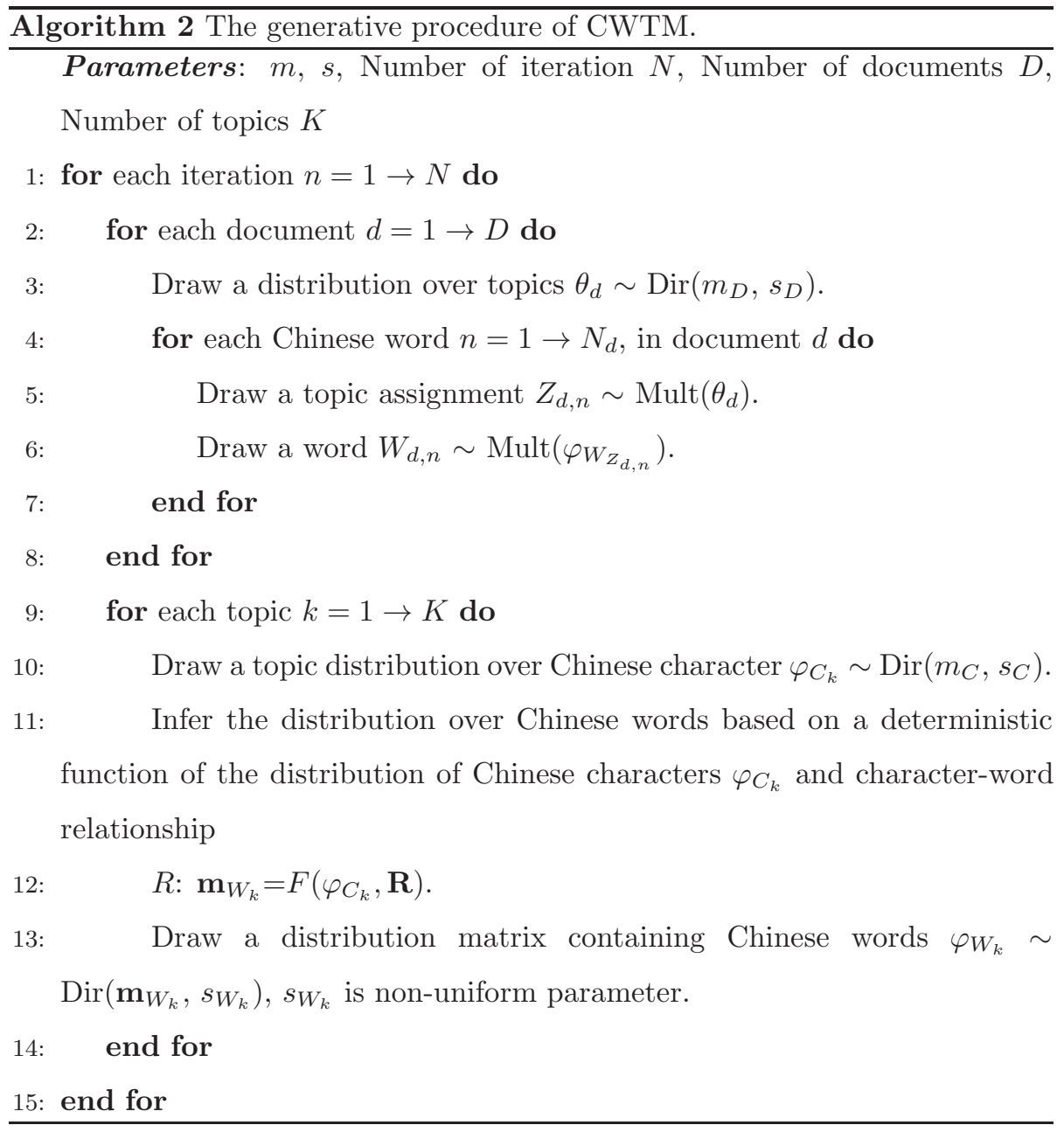

symmetric Dirichlet prior. While CWTM impose each topic-word distribution $\varphi_{W_{k}}$ with a unique asymmetric Dirichlet prior parameterized by $\mathbf{m}_{W_{k}}$ and $s_{W}$ (Wallach et al., 2009). This means that each $\varphi_{W_{k}}$ has a corresponding prior $\operatorname{Dir}\left(\mathbf{m}_{W_{k}}, s_{W}\right)$. And the mean of this Dirichlet prior $-\mathbf{m}_{W}$ is obtained by a deterministic function $\mathrm{F}$, which takes character-word relation $\mathbf{R}$ and topic-character distribution $\varphi_{C_{k}}$ as inputs. Owing to the deterministic characteristic of generating $\varphi_{W_{k}}$ from $\mathbf{R}$ and $\varphi_{C_{k}}, K$ topic-character distributions $\varphi_{C_{1}}, \varphi_{C_{2}}, \ldots, \varphi_{C_{K}}$ should be different to ensure that topic-word distributions $\varphi_{W_{(1: K)}}$ own priors with different mean parameters $\mathbf{m}_{W_{(1: K)}}$. Therefore, the 
character-word relation is incorporated as prior, which could make it less sensitive to errors caused by character-word relation, rather than being hardcoded into the model (Petterson et al., 2010). And the balance between the prior originated from character-word relation and observed data could be controlled by adjusting the Dirichlet precision parameter $-s_{W}$.

\section{Posterior Inference}

Several methods have been proposed to do inference in LDA-like topic modeling. In this paper, we employ Gibbs sampling (Griffiths and Steyvers, 2004; Steyvers and Griffiths, 2007), which can be regarded as a special form of Markov Chain Monte Carlo method (Bishop, 2006; Gilks et al., 1996).

\subsection{Document Likelihood}

As illustrated in the graphical model Fig. 6(b) we can see the joint distribution $p\left(\mathbf{W}, \mathbf{Z} \mid m_{D}, s_{D}, \mathbf{m}_{W}, s_{W}\right)$ which could be factored as below:

$$
p\left(\mathbf{W}, \mathbf{Z} \mid m_{D}, s_{D}, \mathbf{m}_{W}, s_{W}\right)=p\left(\mathbf{W} \mid \mathbf{Z}, \mathbf{m}_{W}, s_{W}\right) p\left(\mathbf{Z} \mid m_{D}, s_{D}\right)
$$

By eliminating $\varphi_{W}$ and $\theta$, respectively, the two terms on the right-hand side of above equation can be obtained:

$$
\begin{array}{r}
p\left(\mathbf{W} \mid \mathbf{Z}, \mathbf{m}_{W}, s_{W}\right)=\int p\left(\mathbf{W} \mid \mathbf{Z}, \varphi_{W}\right) p\left(\varphi_{W} \mid \mathbf{m}_{W}, s_{W}\right) d \mathbf{m}_{W} \\
=\prod_{k=1}^{K} \frac{\Gamma\left(s_{W}\right)}{\Gamma\left(s_{W}+n_{k}^{T}\right)} \prod_{i=1}^{V_{w}} \frac{\Gamma\left(n_{k, i}^{T W}+s_{W} * \mathbf{m}_{W_{k, i}}\right)}{\Gamma\left(s_{W} * \mathbf{m}_{W_{k, i}}\right)} \\
p\left(\mathbf{Z} \mid m_{D}, s_{D}\right)=\int p(\mathbf{Z} \mid \theta) p\left(\theta \mid m_{D}, s_{D}\right) d \theta \\
=\prod_{d=1}^{N} \frac{\Gamma\left(s_{D}\right)}{\Gamma\left(s_{D}+n_{d}^{D}\right)} \prod_{k=1}^{K} \frac{\Gamma\left(n_{d, k}^{D T}+s_{D} * m_{D_{k}}\right)}{\Gamma\left(s_{D} * m_{D_{k}}\right)}
\end{array}
$$

where $n^{T W}$ is a matrix containing the counts of the number of times each Chinese word is assigned to each topic and $n^{D T}$ is the count number of Chinese words that are assigned to each topic in each document, say, $n_{k, i}^{T W}$ denotes 
the count number of $i$ th Chinese word in vocabulary is assigned to topic $k$. Analogously, $n_{d, k}^{D T}$, element of $n^{D T}$, means the count number of Chinese words which are assigned to topic $k$ in document $d$. And also:

$$
n_{k}^{T}=\sum_{i=1}^{V_{w}} n_{k, i}^{T W}, \quad n_{d}^{D}=\sum_{k=1}^{K} n_{d, k}^{D T}
$$

\subsection{Gibbs Sampling}

To perform Gibbs sampling, we need a sampler $p\left(Z_{d, n}=k \mid Z_{d, n-}, \mathbf{W}\right)$ from which new samples are drawn. $n$ means the $n$th iteration. The subscript '' in $Z_{d, n-}$ represents that current token indexed by $(d, n)$ is not taken into consideration. This sampler is similar to that of standard LDA proposed by Griffiths and Steyvers in (Griffiths and Steyvers, 2004). And the derivation of standard LDA's inference algorithm based on Gibbs sampling (Heinrich, 2009).

$$
p\left(Z_{d, n}=k \mid Z_{d, n-}, \mathbf{W}\right) \propto \frac{\left[n_{k, v-}^{T W}+\mathbf{m}_{W_{k, v}}\right]\left[n_{d, k-}^{D T}+s_{d} * m_{D_{k}}\right]}{\sum_{i=1}^{V_{w}} n_{k, i-}^{T W}+s_{W}}
$$

Noting $\mathbf{W}=\left\{W_{d, n}=v, W_{d, n-}\right\}, n_{k, v-}, n_{d, k-}$ and $n_{k, i-}$ are consistent with $Z_{d, n-}$ in the same iteration.

As this Gibbs sampling method directly estimate $Z_{d, n}$ for each word in the corpus, topic-word distributions $\varphi_{W}$ and document-topic distributions $\theta$ can be obtained as follow:

$$
\begin{gathered}
\varphi_{W_{k, i}}=\frac{n_{k, i}^{T W}+s_{W} * \mathbf{m}_{W_{k, i}}}{\sum_{i=1}^{V_{w}} n_{k, i}^{T W}+s_{W}} \\
\theta_{d, k}=\frac{n_{d, k}^{D T}+s_{\theta} * m_{\theta_{k}}}{\sum_{k=1}^{K} n_{d, k}^{D T}+s_{\theta}}
\end{gathered}
$$

\subsection{Estimate Characters Topic Distribution}

As illustrated in Section 3, the $\mathbf{m}_{W}$ is regarded as unknown, which is computed from topics over Chinese characters through a deterministic process by the function $F\left(\varphi_{C}, \mathbf{R}\right)$. Therefore it is necessary to estimate topics over Chinese characters. 
Here we assume that each Chinese character contained in a Chinese word token $W_{d, n}$ in the corpus is directly sampled from the corresponding charactertopic multinomial distribution for approximately estimate $\varphi_{C}$ :

$$
C_{d, n}^{(j)} \sim \operatorname{Mult}\left(\varphi_{C_{Z_{d, n}}}\right)
$$

where $j$ denotes the index of the character in the word token $W_{d, n}$.

This means all characters in a word shared the identical topic, which is the same as the word's topic assignment. For example, if the Chinese word " $i i$ suan" $\left(W_{d, n}\right)$ is assigned with topic $k$, then its component characters " $j i "$ $\left(C_{d, n}^{(1)}\right)$ and "suan" $\left(C_{d, n}^{(2)}\right)$ are assigned the same topic $k$. This makes sense because characters in a word are inclined to express more related meanings.

Thus, the topic-character distribution could be approximately estimated as:

$$
\varphi_{C_{k, j}}=\frac{n_{k, j}^{T C}+s_{C} * m_{C_{k, j}}}{\sum_{j=1}^{V_{c}} n_{k, j}^{T C}+s_{C}}
$$

where $n_{k, j}^{T C}$ denotes the count number of Chinese characters indexed by $j$ in character vocabulary is assigned to topic $k$.

SA-CWTM has the following hyperparameters: $m_{D}, s_{D}, m_{C}, s_{C}, s_{W}$, which are either Dirichlet mean parameter or Dirichlet precision parameter. As with the standard LDA model, we use symmetric priors in the latter experiment section. That is to say, we set $m_{D}$ and $m_{C}$ as uniform distributions. For the other Dirichlet precision parameter, that setting to fix $s_{D}=50, s_{W}=$ $V_{w} * 0.1$ and $s_{C}=V_{c} * 0.1$ for all number of topics based on empirical research in (Griffiths and Steyvers, 2004).

\section{Experimental Studies}

In this section, we conduct a series of experiments on two datasets, NEWS1W from Sogou laboratory and Ch-wikipedia Dataset from Chinese Wikipedia ${ }^{4}$ to compare the performance of word-based LDA, character-based LDA and

\footnotetext{
${ }^{4}$ http://zh.wikipedia.org
} 
the novel CWTM in text modeling and classification. The model inference is based on Gibbs Sampling and implementation of the model is based on Gibb$\operatorname{sLDA}++^{5}$.

\subsection{Database and Preprocessing}

Ch-Wikipedia Dataset ${ }^{6}$ Ch-Wikipedia Dataset is a Chinese corpus consists 3103 documents from 9 categories listed in Table 2. $90 \%$ of the documents are randomly chosen as the training set and the remaining for test. The documents in this corpus are crawled from a collection of articles in Chinese Wikipedia, which is one of the biggest internet information websites in Chinese language. There are 20 classes in original corpus that cover literature, media, sports, politics and other topics. Each article is split into some parts by section heading. The texts containing less than 100 Chinese characters will be ignored in our research. Topics of some classes are similar, such as "humanities" and "culture \& society", which can be merged into one category "culture". On the other hand, some classes containing less than 150 articles are abandoned as well if they can not be merged to a certain category. We list the final categories in Table 2 after merging similar ones manually. When we do the Chinese character-based topic modeling, we remove the rare characters that appear less than 3 times across the whole corpus and the terms appearing in over $50 \%$ throughout the whole documents which we consider as stop words (Zhao et al., 2011b). When modeling for word-based topics, we first get a "stopwords list" and ignore them when constructing the vocabulary. Words appear less than 3 times through all documents are removed as well. The Chinese words are segmented from documents by using ICTCLAS- $09^{7}$ in our experiments. After the preprocessing we get 48506 unique Chinese words and 4825 unique Chinese

\footnotetext{
${ }^{5} \mathrm{C} / \mathrm{C}++$ Implementation of Latent Dirichlet Allocation.

http://gibbslda.sourceforge.net/

${ }^{6} \mathrm{Ch}$-Wikipedia Dataset is available at: http://icmll.buaa.edu.cn/zh_wikipedia

${ }^{7}$ ICTCLAS is an integrate Chinese lexical analysis system which provide a tool for Chinese word segmentation. http://ictclas.org/.
} 
Table 2: Summary of the Ch-Wikipedia Dataset, the articles are extracted from Chinese Wikipedia website.

\begin{tabular}{c|ccc}
\hline Category & Training & Test & Total \\
\hline Culture & 285 & 71 & 356 \\
Biology \& Medicine & 327 & 82 & 409 \\
Natural Science & 279 & 70 & 349 \\
Geography & 374 & 93 & 467 \\
History & 424 & 106 & 530 \\
Traffic & 156 & 39 & 195 \\
Warfare \& Military & 206 & 52 & 258 \\
Scholar \& Occupational Figures & 145 & 36 & 181 \\
Political \& Military Figures & 286 & 72 & 358 \\
\hline
\end{tabular}

characters.

NEWS1W Another Chinese corpus we used is a news archive for classification provided by Sogou laboratory ${ }^{8}$. The documents in this corpus are collected from the website of Sohu ${ }^{9}$ news, which were manually edited and classified into 10 classes with contents covered military, education, tourism, culture, IT, job, auto, sports, finance and health. Each class contains 8000 documents. But few of these documents contain nothing but some meaningless non-Chinese symbols. Therefore those documents contain less than 100 Chinese characters will be ignored in our research. We selected 10000 documents with 1000 documents per class from the corpus as our experiment data, which is denoted by NEWS1W ${ }^{10}$. Then we implement the above preprocessing step the same as Ch-Wikipedia Dataset. After that, NEWS1W contains 25673 unique Chinese words and 4142 unique Chinese characters. In the experiment, $10 \%$ of the documents in NEWS1W were held out as test set and the remaining $90 \%$ documents

\footnotetext{
${ }^{8}$ Sogou news classification corpus, http://www.sogou.com/labs/dl/c.html

${ }^{9} \mathrm{Sohu}$ is one of the China's biggest Internet media companies, website: www.sohu.com

10 http://icmll.buaa.edu.cn/projects/topicmodel/dataset/
} 
were used to train topic models based on characters and words with different numbers of topics.

\subsection{Experiments for Topic Extraction}

Judging the interpretability of a set of topics is highly subjective. A concept called "word intrusion" was introduced by Chang et al in (Chang et al., 2009). In the paper, an "intruding" word from a set of words is determined when it does not semantically fit with other words, and "topic intrusion" is determined when the learned document-topic portion $\theta_{d}$ does not appropriately describe the semantic theme of the document. To testified the topic interpretability of CWTM, we are mostly interested in subjective exhibiting the low incidence of "word intrusion".

Fig. 7 shows two topics from Ch-Wikipedia and NEWS1W, respectively, manually labeled as "Sports" learned from the NEWS1W and "Natural Science" from Ch-Wikipedia. The number of the topic is set $K=50$. We demonstrate the top 10 words independently extracted from CWTM, word-based LDA and character-based LDA from the specific topics. It is obviously that the cohesiveness terms with high probabilities are in keeping with the interpretability of the topic. Words with high probability are assign to the topic as one would expect to have high importance.

Except for the interpretability of topics, another important factor to judge the model is the robustness for out-of-vocabulary words As we mentioned in Section 1, for the words not appeared in the training data, characters composing these words have appeared as component of other words in the training data. Fig. 8 displays the top 10 unseen words in both training corpuses: NEWS1W and Ch-Wikipedia. There are 147 unseen words in NEWS1W and 6410 unseen words in Ch-Wikipedia. They are ranked by their frequency of occurrence in test set.

In Fig. 9 and 10 , we choose three frequent topics from the two corpuses: "Sports" "Health" and "IT" from NEWS1W and "Natural Science" "Warfare \& Military" and "Traffic" from Ch-Wikipedia. For each topic, we select 10 
Figure 7: Topic samples extracted from CWTM, word-based LDA and character-based LDA on two corpuses: NEWS1W and Ch-Wikipedia.

\begin{tabular}{c|c}
\hline NEWS1W & Ch-Wikipedia \\
\hline 哈根达斯(Haagen-Dazs) & 远足者(Tripper) \\
主客场(Home and Away) & 宣传画(Posters) \\
人机(Man-machine) & 磁力线(Magnetic line) \\
绑腿(Puttee) & 娘子军(Women soldiers) \\
水痘(Chicken pox) & 腰椎(Lumbar) \\
拾荒(Pepenador) & 骈文(Parallel prose) \\
长征四号(Long march 4) & 墙砖(Wall brick) \\
龙门(Dragon Gate) & 维权(Safeguard legal rights) \\
南平市(Nanping city) & 微调(Fine tuning) \\
护肩(Sholder) & 收市(closing quotation) \\
\hline
\end{tabular}

Figure 8: Top unseen words in training corpuses of NEWS1W and Ch-Wikipedia. 


\begin{tabular}{c|cc|cc}
\hline \multicolumn{5}{|c}{ NEWS1W } \\
\hline Word & CWTM & Topic & Word-based LDA & Topic \\
\hline $\begin{array}{c}\text { 主客场 } \\
\text { (Home and Away) }\end{array}$ & $0.0044(450)$ & $\begin{array}{c}\text { Sports } \\
\text { (Topic 6) }\end{array}$ & - & - \\
\hline $\begin{array}{c}\text { 德国队 } \\
\text { (German team) }\end{array}$ & $0.0032(456)$ & $\begin{array}{c}\text { Sports } \\
\text { (Topic 6) }\end{array}$ & $0.0009(646)$ & $\begin{array}{c}\text { Culture } \\
\text { (Topic 23) }\end{array}$ \\
\hline $\begin{array}{c}\text { 大胜 } \\
\text { (Victory) }\end{array}$ & $0.0031(468)$ & $\begin{array}{c}\text { Sports } \\
\text { (Topic 6) }\end{array}$ & $0.001(133)$ & $\begin{array}{c}\text { Military } \\
\text { (Topic 51) }\end{array}$ \\
\hline $\begin{array}{c}\text { 发球 } \\
\text { (Launch ball) }\end{array}$ & $0.0031(477)$ & $\begin{array}{c}\text { Sports } \\
\text { (Topic 6) }\end{array}$ & $0.0003(1614)$ & $\begin{array}{c}\text { Sports } \\
\text { (Topic 11) }\end{array}$ \\
\hline $\begin{array}{c}\text { 接球 } \\
\text { (Catch ball) }\end{array}$ & $0.0031(532)$ & $\begin{array}{c}\text { Sports } \\
\text { (Topic 6) }\end{array}$ & $0.0014(352)$ & $\begin{array}{c}\text { Sports } \\
\text { (Topic 11) }\end{array}$ \\
\hline $\begin{array}{c}\text { 国奥队 } \\
\text { (Olympic team) }\end{array}$ & $0.0012(680)$ & $\begin{array}{c}\text { Sports } \\
\text { (Topic 6) }\end{array}$ & $0.0008(517)$ & $\begin{array}{c}\text { Sports) } \\
\text { (Topic 11) }\end{array}$ \\
\hline $\begin{array}{c}\text { 对攻 } \\
\text { (Compound Attack) }\end{array}$ & $0.0011(736)$ & $\begin{array}{c}\text { Sports } \\
\text { (Topic 6) }\end{array}$ & $0.0007(775)$ & $\begin{array}{c}\text { Military } \\
\text { (Topic 51) }\end{array}$ \\
\hline $\begin{array}{c}\text { 赛道 } \\
\text { (Speedway) }\end{array}$ & $0.0011(792)$ & $\begin{array}{c}\text { Sports } \\
\text { (Topic 6) }\end{array}$ & $0.0041(106)$ & $\begin{array}{c}\text { Sports } \\
\text { (Topic 11) }\end{array}$ \\
\hline $\begin{array}{c}\text { 女足 } \\
\text { (Women football) }\end{array}$ & $0.0005(880)$ & $\begin{array}{c}\text { Sports } \\
\text { (Topic 6) }\end{array}$ & $0.0001(979)$ & $\begin{array}{c}\text { Sports } \\
\text { (Topic 11) }\end{array}$ \\
\hline 国奥 \\
(Olympic)
\end{tabular}

(a) Topic of Sport (NEWS1W)

\begin{tabular}{|c|c|c|c|c|}
\hline \multicolumn{5}{|c|}{ NEWS1W } \\
\hline Word & CWTM & Topic & Word-based LDA & Topic \\
\hline 关节炎 & $0.0399(60)$ & Health & $0.0097(328)$ & Health \\
\hline (Arthritis) & & (Topic 11) & & (Topic 24) \\
\hline 体液 & $0.0194(129)$ & Health & $0.0083(446)$ & Health \\
\hline (Body fluid) & & (Topic 11) & & (Topic 24) \\
\hline 核磁共振 & $0.017(237)$ & Health & $0.0071(632)$ & Health \\
\hline (Nuclear magnetic resonance) & & (Topic 11) & & (Topic 24) \\
\hline 溶液 & $0.0115(306)$ & Health & $0.0013(614)$ & Education \\
\hline (Solution) & & (Topic 11) & & (Topic 50) \\
\hline 皮脂腺 & $0.0104(323)$ & Health & $0.0004(1532)$ & Health \\
\hline (Sebaceous gland) & & (Topic 11) & & (Topic 24) \\
\hline 息肉 & $0.0091(445)$ & Health & $0.0004(1606)$ & Health) \\
\hline (Polyp) & & (Topic 11) & & (Topic 24) \\
\hline 水痘 & $0.0074(483)$ & Health & - & - \\
\hline (Chicken pox) & & (Topic 11) & & \\
\hline 偏方 & $0.0069(495)$ & Health & $0.0074(509)$ & Health \\
\hline (Folk remedy) & & (Topic 11) & & (Topic 24) \\
\hline 骨架 & $0.0057(545)$ & Health & $0.0061(891)$ & Health \\
\hline (Skeleton) & & (Topic 11) & & (Topic 24) \\
\hline 卫生 & $0.0044(615)$ & Health & $0.00001(1580)$ & Health \\
\hline (Sanitation) & & (Topic 11) & & (Topic 24) \\
\hline
\end{tabular}

(b) Topic of Health (NEWS1W)

\begin{tabular}{|c|c|c|c|c|}
\hline \multicolumn{5}{|c|}{ NEWS1W } \\
\hline Word & CWTM & Topic & Word-based LDA & Topic \\
\hline $\begin{array}{c}\text { 技术 } \\
\text { (Technology) }\end{array}$ & $0.0203(21)$ & $\begin{array}{c}\text { IT } \\
\text { (Topic 17) }\end{array}$ & $0.0078(268)$ & $\begin{array}{c}\text { Auto } \\
\text { (Topic 33) }\end{array}$ \\
\hline $\begin{array}{c}\text { 下载 } \\
\text { (Download) }\end{array}$ & $0.0071(168)$ & $\begin{array}{c}\text { IT } \\
\text { (Topic 17) }\end{array}$ & $0.0076(331)$ & $\begin{array}{c}\text { IT } \\
\text { (Topic 9) }\end{array}$ \\
\hline $\begin{array}{c}\text { 五子棋 } \\
\text { (Gomoku) }\end{array}$ & $0.0068(54)$ & $\begin{array}{c}\text { IT } \\
\text { (Topic 17) }\end{array}$ & $0.0061(464)$ & $\begin{array}{c}\text { IT } \\
\text { (Topic 9) }\end{array}$ \\
\hline $\begin{array}{l}\text { 主机 } \\
\text { (Host) }\end{array}$ & $0.0028(278)$ & $\begin{array}{c}\text { IT } \\
\text { (Topic 17) }\end{array}$ & $0.00009(1061)$ & $\begin{array}{c}\text { IT } \\
\text { (Topic 9) }\end{array}$ \\
\hline $\begin{array}{c}\text { 传出 } \\
\text { (Come out) }\end{array}$ & $0.0012(316)$ & $\begin{array}{c}\text { IT } \\
\text { (Topic 17) }\end{array}$ & $0.0014(519)$ & $\begin{array}{c}\text { Culture } \\
\text { (Topic 23) }\end{array}$ \\
\hline $\begin{array}{c}\text { 人机 } \\
\text { (Man-machine) }\end{array}$ & $0.001(370)$ & $\begin{array}{c}\text { IT } \\
\text { (Topic 17) }\end{array}$ & & \\
\hline $\begin{array}{c}\text { 估算 } \\
\text { (Estimate) }\end{array}$ & $0.0009(381)$ & $\begin{array}{c}\text { IT } \\
\text { (Topic 17) }\end{array}$ & $0.0072(407)$ & $\begin{array}{l}\text { Education } \\
\text { (Topic 50) }\end{array}$ \\
\hline $\begin{array}{c}\text { 可行 } \\
\text { (Feasible) }\end{array}$ & $0.0007(428)$ & $\begin{array}{c}\text { IT } \\
\text { (Topic 17) }\end{array}$ & $0.0043(995)$ & $\begin{array}{c}\text { IT } \\
\text { (Topic 9) }\end{array}$ \\
\hline $\begin{array}{c}\text { 互联 } \\
\text { (Interconnection) }\end{array}$ & $0.0005(496)$ & $\begin{array}{c}\text { IT } \\
\text { (Topic 17) }\end{array}$ & $0.00001(2227)$ & $\begin{array}{c}\text { IT } \\
\text { (Topic 9) }\end{array}$ \\
\hline $\begin{array}{c}\text { 发布会 } \\
\text { (Conference) }\end{array}$ & $0.0004(530)$ & $\begin{array}{c}\text { IT } \\
\text { (Topic 17) }\end{array}$ & $0.0001(1058)$ & $\begin{array}{c}\text { IT } \\
\text { (Topic 9) }\end{array}$ \\
\hline
\end{tabular}

(c) Topic of IT (NEWS1W)

Figure 9: Some representational words extracted from 3 sampled topics learned by CWTM and word-based LDA. The unseen words learned by CWTM are in bold font. 


\begin{tabular}{|c|c|c|c|c|}
\hline \multicolumn{5}{|c|}{ Ch-Wikipedia } \\
\hline Word & CWTM & Topic & Word-based LDA & Topic \\
\hline 主战场 & $0.0561(122)$ & Warfare \& Military & $0.0025(1551)$ & Warfare \& Military \\
\hline (Main battlefeleld) & & (Topic 2) & & (Topic 91) \\
\hline $\begin{array}{l}\text { 伤装 } \\
\end{array}$ & $0.0468(230)$ & Warfare \& Military & $0.0057(436)$ & Culture \\
\hline (pretend) & & (Topic 2) & & (Topic 47) \\
\hline 会战 & $0.0393(241)$ & Warfare \& Military & $0.0197(67)$ & Warfare \& Military \\
\hline (Pitched battle) & & (Topic 2) & & (Topic 27) \\
\hline 举兵 & $0.0299(351)$ & Warfare \& Military & $0.0075(297)$ & History \\
\hline (Raise army) & & (Topic 2) & & (Topic 30) \\
\hline 册封 & $0.0281(357)$ & Warfare \& Military & $0.0227(80)$ & Culture \\
\hline (Canonize) & & (Topic 2) & & (Topic 47) \\
\hline 殉难 & $0.0262(362)$ & Warfare \& Military & $0.0043(720)$ & History \\
\hline (Martyrdom) & & (Topic 2) & & (Topic 30) \\
\hline 五四运动 & $0.0168(381)$ & Warfare \& Military & $0.0035(688)$ & Warfare \& Military \\
\hline (May 4th movement) & & (Topic 2) & & (Topic 27) \\
\hline 步兵团 & $0.0094(418)$ & Warfare \& Military & $0.0211(78)$ & Warfare \& Military \\
\hline (Infantry) & & (Topic 2) & & (Topic 27) \\
\hline 㪴灭战 & $0.0056(454)$ & Warfare \& Military & $0.0047(637)$ & Warfare \& Military \\
\hline (War of ammihilation) & & (Topic 2) & & (Topic 27) \\
\hline 娘子军 & $0.0037(538)$ & Warfare \& Military & & \\
\hline (Women soldiers) & & (Topic 2) & & \\
\hline
\end{tabular}

(a) Topic of Natural Science (Ch-Wikipedia) (b) Topic of War. \& Military (Ch-Wikipedia)

\begin{tabular}{|c|c|c|c|c|}
\hline \multicolumn{5}{|c|}{ Ch-Wikipedia } \\
\hline Word & CWTM & Topic & Word-based LDA & Topic \\
\hline $\begin{array}{c}\text { 交通量 } \\
\text { (Car traffic volume) }\end{array}$ & $0.0563(33)$ & $\begin{array}{c}\text { Traffic } \\
\text { (Topic 33) }\end{array}$ & $0.000013(2024)$ & $\begin{array}{c}\text { Traffic } \\
\text { (Topic } 14\end{array}$ \\
\hline $\begin{array}{c}\text { 东站 } \\
\text { (East station) }\end{array}$ & $0.0375(48)$ & $\begin{array}{c}\text { Traffic } \\
\text { (Topic 33) }\end{array}$ & $0.0083(270)$ & $\begin{array}{c}\text { Traffic } \\
\text { (Topic 14 } \\
\end{array}$ \\
\hline $\begin{array}{l}\text { 润滑油 } \\
\text { (Lube) }\end{array}$ & $0.0281(59)$ & $\begin{array}{c}\text { Traffic } \\
\text { (Topic 33) }\end{array}$ & $0.00001(2009)$ & $\begin{array}{r}\text { Traffic } \\
\text { (Topic 14 } \\
\end{array}$ \\
\hline $\begin{array}{c}\text { 地铁站 } \\
\text { (Subway station) }\end{array}$ & $0.0134(92)$ & $\begin{array}{c}\text { Traffic } \\
\text { (Topic 33) } \\
\end{array}$ & $0.0083(254)$ & $\begin{array}{r}\text { Traffic } \\
\text { (Topic 14 }\end{array}$ \\
\hline $\begin{array}{c}\text { 方向盘 } \\
\text { (Steering wheel) }\end{array}$ & $0.0067(122)$ & $\begin{array}{c}\text { Traffic } \\
\text { (Topic 33) }\end{array}$ & $0.00001(2053)$ & $\begin{array}{r}\text { Traffic } \\
\text { (Topic } 14 \\
\end{array}$ \\
\hline $\begin{array}{c}\text { 导航仪 } \\
\text { (Avigraph) }\end{array}$ & $0.0054(131)$ & $\begin{array}{c}\text { Traffic } \\
\text { (Topic 33) }\end{array}$ & $0.00001(2201)$ & $\begin{array}{r}\text { Traffic } \\
\text { (Topic 14 } \\
\end{array}$ \\
\hline $\begin{array}{c}\text { 机动 } \\
\text { (Engine driven) }\end{array}$ & $0.0027(201)$ & $\begin{array}{c}\text { Traffic } \\
\text { (Topic 33) }\end{array}$ & $0.00001(2101)$ & $\begin{array}{c}\text { Traffic } \\
\text { (Topic 14 } \\
\end{array}$ \\
\hline $\begin{array}{c}\text { 磁力线 } \\
\text { (Magnetic line) }\end{array}$ & $0.0019(305)$ & $\begin{array}{c}\text { Traffic } \\
\text { (Topic 33) }\end{array}$ & - & - \\
\hline $\begin{array}{c}\text { 爆胎 } \\
\text { (Tire burst) }\end{array}$ & $0.0013(310)$ & $\begin{array}{c}\text { Traffic } \\
\text { (Topic 33) } \\
\end{array}$ & $0.00001(2067)$ & $\begin{array}{r}\text { Traffic } \\
\text { (Topic 14 } \\
\end{array}$ \\
\hline $\begin{array}{c}\text { 发现号 } \\
\text { (Discovery) }\end{array}$ & $0.001(476)$ & $\begin{array}{c}\text { Traffic } \\
\text { (Topic 33) }\end{array}$ & $0.00001(1854)$ & $\begin{array}{c}\text { Traffic } \\
\text { (Topic } 14\end{array}$ \\
\hline
\end{tabular}

(c) Topic of Traffic (Ch-Wikipedia)

Figure 10: Some representational words extracted from 3 sampled topics learned by CWTM and word-based LDA. The unseen words learned by CWTM are in bold font. 
representational words associated with the probability $P(w \mid t)$ it belongs to this topic and its ranking. For example, the 2nd term in Fig. 9(a) "German team", its word probability is 0.0032 and it ranks 456 in the topic of Sport, the 6th topic learned by CWTM on corpus NEWS1W. Comparing to the word-based LDA, this term ranks 646 with probability of 0.0009 , however, it is in the topic of "Culture" when learned by the word-based LDA. For unseen terms such as "Home and Away", it can be learned by CWTM but it has no corresponding probability in the word-based LDA. It's obviously that, some of the words relevant to the topics, even not appeared in the training data listed in Fig. 8, were assigned relatively higher probabilities in corresponding topics by CWTM. The distributed probabilities for common words is higher than that obtained from Word-based LDA.

The goodness of topic extraction is hard to measure as it is very subjective. In this paper, we were trying to explore if we can assign fair probabilities to unseen Chinese words. Though the word is unseen, if its consisting Chinese characters are with high probability in this topic, then this unseen word is also with thigh probability for the semantic correlation between them. For example, the word "zhu ke chang" (home and away), though never appeared in training documents in NEWS1W, its component characters "zhu", "ke", and "chang" are the components of those words appeared in training data. Therefore, it obtained a higher probability in CWTM. On the other hand, not surprisingly, none of these words were ranked into top 1000 words in the corresponding topic extracted by standard LDA model.

\subsection{Experiments of Document Classification}

In this experiment, we employ the Support Vector Machines(SVM) to classify the documents in the two corpus. The word-based LDA, character-based LDA and CWTM are trained separately. The result of $\theta$ is adopted as the feature for text classification. The format of the feature is showed in Table 3. Each text is represented as a $1 \times K$ vector with float data. Every feature means a 
Table 3: The details of features for text classification when topic number is 100 .

\begin{tabular}{c|cccc}
\hline Document & \multicolumn{5}{|c}{ Feature Data (100 dimension) } \\
\hline$D 1$ & 0.003876 & 0.034884 & $\ldots$ & 0.011628 \\
$D 2$ & 0.006219 & 0.001244 & $\ldots$ & 0.028607 \\
$D 3$ & 0.004464 & 0.022321 & $\ldots$ & 0.013393 \\
$D 4$ & 0.011628 & 0.002326 & $\ldots$ & 0.006977 \\
$D 5$ & 0.004202 & 0.088235 & $\ldots$ & 0.012605 \\
$D 6$ & 0.001089 & 0.003268 & $\ldots$ & 0.022876 \\
$D 7$ & 0.015581 & 0.004249 & $\ldots$ & 0.035411 \\
$D 8$ & 0.002 & 0.014 & $\ldots$ & 0.05 \\
\hline
\end{tabular}

topic probability distribution over a document. We adopted libsvm ${ }^{11}$ in our experiments. It's showed in Table 4 that the classifiers with RBF kernel have the highest accuracy rate on each model when set the topic number to 100 for the LDA to do classification both on Ch-wikipedia and NEWS1W. Thus in the following experiments, we use libsvm with RBF kernel for category prediction. The accuracy of the text classification is measured by the precision of document classification. In addition, the classification results of the four different kinds of CWTM models are also shown in Fig. 11 and Fig. 12 to verify that the SA-CWTM treated as the final CWTM has the best performance. Fig. 11 is the results on Ch-wikipedia. The SA-CWTM model get the highest accuracy is $76.8 \%$ when topic number is set to 200. Fig. 12 is the results on NEWS1W. The highest result is $89.1 \%$ when topic number is set to 100 on SA-CWTM. It is obviously that the SA-CWTM which we prefer as the final CWTM model achieve the best classification accuracy on both two corpus.

We apply libsvm to train a model using the training data which was reduced to the dimension of number of topics and predict the remaining test

\footnotetext{
${ }^{11}$ LIBSVM is an integrated software for support vector classification, regression and distribution estimation. http://www.csie.ntu.edu.tw/ cjlin/libsvm/.
} 
Table 4: Accuracy rate of SVM classifiers with different kernels on two corpus when set the topic number to 100 .

\begin{tabular}{|c|c|c|c|c|}
\hline Database & \multicolumn{2}{|c|}{ Linear Polynomial } & \multicolumn{2}{|c|}{ RBF Sigmoid } \\
\hline \multicolumn{5}{|l|}{ Ch-Wikipedia } \\
\hline Word-based training texts & 0.642 & 0.548 & 0.817 & 0.627 \\
\hline Word-based test texts & 0.654 & 0.385 & 0.628 & 0.668 \\
\hline Character-based training texts & 0.820 & 0.639 & 0.989 & 0.776 \\
\hline Character-based test texts & 0.712 & 0.533 & 0.725 & 0.704 \\
\hline CWTM training texts & 0.821 & 0.573 & 0.797 & 0.746 \\
\hline CWTM test texts & 0.726 & 0.557 & 0.728 & 0.709 \\
\hline Average & 0.729 & 0.539 & 0.781 & 0.705 \\
\hline \multicolumn{5}{|l|}{ NEWS1W } \\
\hline Word-based training texts & 0.649 & 0.554 & 0.811 & 0.622 \\
\hline Word-based test texts & 0.86 & 0.688 & 0.826 & 0.818 \\
\hline Character-based training texts & 0.831 & 0.85 & 0.778 & 0.776 \\
\hline Character-based test texts & 0.75 & 0.561 & 0.792 & 0.764 \\
\hline CWTM training texts & 0.818 & 0.641 & 0.891 & 0.883 \\
\hline CWTM test texts & 0.887 & 0.623 & 0.905 & 0.891 \\
\hline Average & 0.799 & 0.653 & 0.834 & 0.792 \\
\hline
\end{tabular}




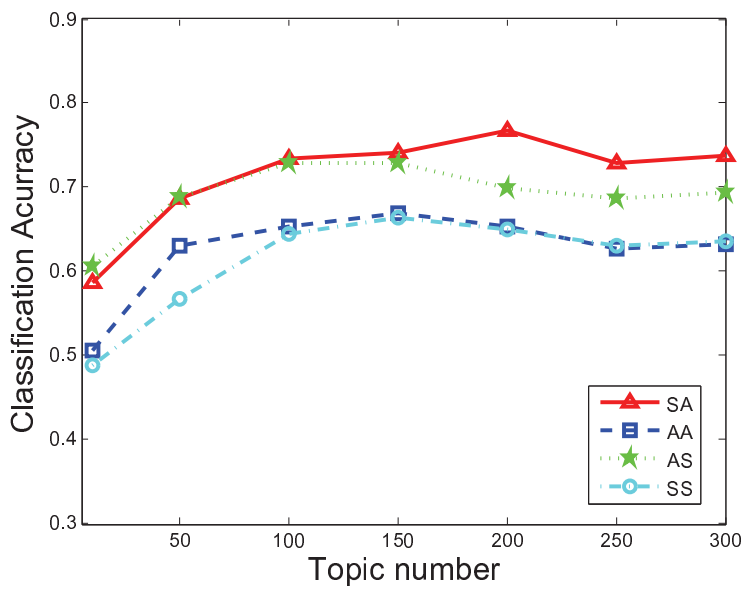

Figure 11: Comparison of documents classification accuracy of the four extended CWTM model on Ch-wikipedia. The SA model get the best performance on average. The highest accuracy is $76.8 \%$ when topic number is set to 200 on SA. So we choose the SA model as the final CWTM.

documents' label. The procedures are repeated with different number of topics varying from 10 to 300 . Fig. 11 illustrates the classification accuracy performance on Ch-wikipedia. For the Ch-wikipedia database, the highest accuracy $76.8 \%$ is performed when the topic number is set to 200 . As for the NEWS1W dataset, the same classification experiments are done by varying the topic number from 10 to 300. The results are showed in Fig. 12. We can see that the SA model get the best accuracy on average. The highest result is $89.1 \%$ when topic number is set to 100 on SA-CWTM. In addition, we can see from the figure that placing an asymmetric Dirichlet prior over either document-topic distribution or topic-word distribution, the classification performance both can be improved absolutely. It is consistent with the conclusion in (Wallach et al., 2009) that asymmetric priors means more natural LDA and achieve better performance. AA and SA have higher classification accuracy than SS and AS. That is to say, placing an asymmetric prior over topic-word distribution makes the model obtain better performance than symmetric priors in our experiment, which means that it's a better representation for each topic-word distribution 


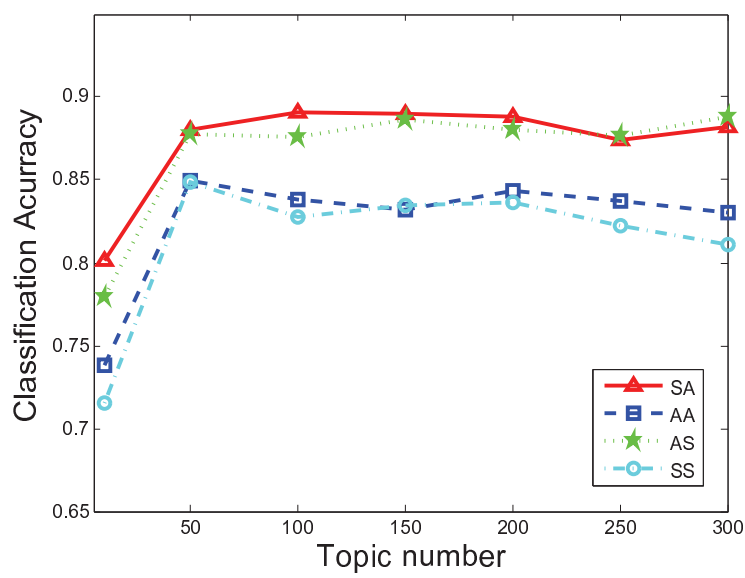

Figure 12: Comparison of documents classification accuracy of the extended four CWTM model on NEWS1W. The SA model obtains the best accuracy on average. The highest result is $89.1 \%$ when topic number is set to 100 on $\mathrm{SA}$.

$\varphi_{W_{k}}$ with a unique asymmetric Dirichlet prior parameterized by $\mathbf{m}_{W_{k}}$ and $s_{W}$ than symmetric after infusing the character-word relationship. Each $\varphi_{W_{k}}$ has a corresponding prior $\operatorname{Dir}\left(\mathbf{m}_{W_{k}}, s_{W}\right)$. And the mean of this Dirichlet prior $\mathbf{m}_{W_{k}}$ is obtained by a deterministic function $\mathrm{F}$.

But the AA model performs similarly with SA model rather than having significantly better performance. It is most likely that when we have removed the stopwords, the words distribution over each topic is more uniform. There are almost no words presenting in nearly all documents. That is, the effect of robustness to skewed word frequency distributions and tend to concentrate stopwords in their own topics is not particularly evident. This is related to the structure of corpus to some extent. So it can get good result even with symmetric prior over document-topic distribution. The figure also shows the trend of the accuracy for all models along with the increment of the topic number. A suitable topic number is good for the classification task. Therefore, an appropriate topic number setting, which may changes with different corpus, can lead better results.

The different classification performances of character-based LDA, word-based 


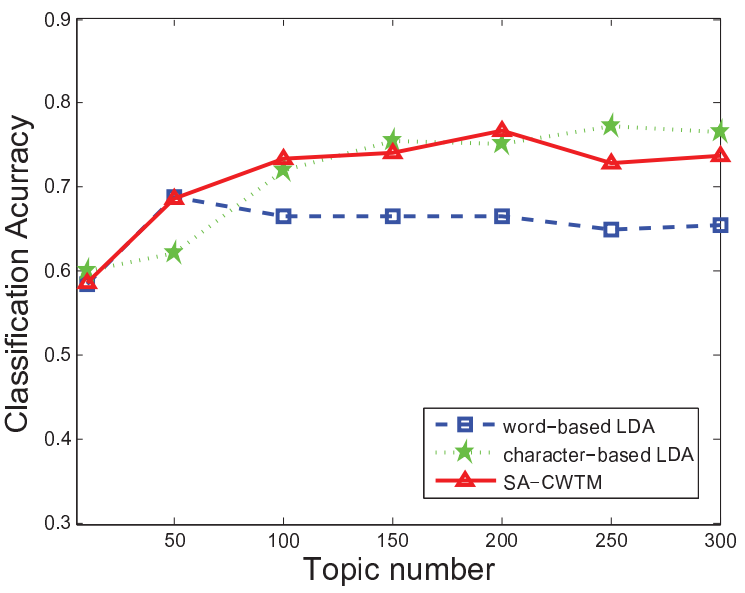

(a) The comparison of character-based LDA, word-based IDA and CWTM on Ch-wikipedia.

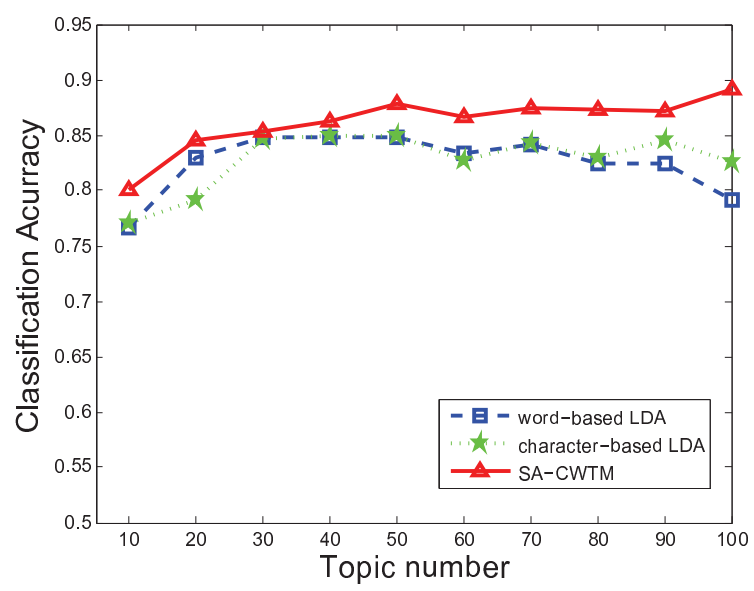

(b) Comparison of documents classification accuracy of character-based LDA, word-based LDA and CWTM model on NEWS1W.

Figure 13: Performance comparison of word-based LDA, character-based LDA and CWTM on two data sets: Ch-wikipedia and NEWS1W. (a) Comparison of the three models on Chwikipedia. CWTM model achieves better results when topic number varies from 10 to 300 . (b) Comparison of documents classification accuracy of the three models on NEWS1W. The CWTM achieves obviously best accuracy comparing to the other two models. 
LDA and SA-CWTM on Ch-wikipedia are showed in Fig. 13(a). It is apparently that SA-CWTM model achieve better result when topic number varies from 10 to 200. But it doesn't have a raise when the topic number is much large up to 300 and more. It is mostly likely because that large topic number bring much sparse document-topic and topic-word distribution by mapping topic-character distribution to topic-word distribution using character-word relationship. The character-based LDA gains better performance instead. The errors brought to the CWTM model by encoding this relation prevent it from performing much better. Thus the setting of topic number has more impacts on CWTM than both character-based and word-based LDA. This may be improved by optimizing the definition of the function $F\left(\varphi_{\mathbf{C}_{k}}, \mathbf{R}\right)$ or a more sophisticated model structure. Fig. 13(b) is the classification results comparison of word-based, character-based LDA and SA-CWTM. The SA-CWTM achieves obviously higher accuracy than the other two models. It performs better at every topic number. Generally, our model outperforms other models in the text classification task.

\section{Conclusions and Future Work}

In this paper, we use a computational approach to study semantic modeling of Chinese language. Latent Dirichlet Allocation, a well-known topic model, is adopted to model Chinese texts based on Chinese words and Chinese characters. Comprehensive empirical results presented on two benchmark data sets, NEWS1W and Ch-Wikipedia, showed that the character-based LDA model outperforms or at least as good as the word-based LDA model in semantics modeling and text classification. Our results suggest that the topic model with Chinese characters can effectively capture the semantic contents in text documents. The computational evidence presented in this paper supports Xu's (Xu, 2001) argument that the Chinese characters, not only the Chinese words, can be used as the basic semantic units in Chinese language modeling.

Though topic models based on Chinese characters achieved competent performance in modeling documents, its limitation is obvious: a considerable num- 
ber of Chinese words have the semantic meaning irrelevant to the meanings of its consisting characters. On the other hand, topic model based on Chinese words did not make use of the connections between those words and their consisting characters. Therefore, we propose a novel topic model by incorporating character-word relation into the topic model by placing an asymmetric prior on the topic-word distribution of the standard LDA. Experiments studies showed that, compared to the word-based LDA and the character-based LDA, the new model can extract more reasonable topics and improve classification performance in given courpuses.

The main contributions of the papers are the following: (1) Besides the superior performance in texts classification, character-based LDA model is more preferable than the word-based model in real-world practice due to its much smaller vocabulary size. (2) By infusing character-word relation into the standard LDA, we proposed a novel topic model which can generate more sensible topics and outperforms both the LDA models based on words and characters, respectively. (3) Placing asymmetric Dirichlet priors over document-topic and topic-word distribution makes the LDA achieve better performance on document classification. (4) Based on comprehensive empirical results, CWTM can learn more interpretable topics and have more robust ability for unseen data (words) than both word-based and character-based LDA.

By considering the character-word relation in topic modeling, we could capture some latent semantic relations between words sharing the same characters, it also brings much noise. It is an interesting topic how could we avoid such noise while keeping the performance. In addition, we can also consider syntactic purposes of words and how important the words order is in texts modeling. This may help us to develop a more structured view of the semantics of a language.

\section{Acknowledgements}

This work was funded by the National Science Foundation of China Nos. 61305047 and 61401012. 


\section{References}

Asuncion, A., Welling, M., Smyth, P., , Teh, Y., 2009. On smoothing and inference for topic models. In: Proceedings of the 25th Conference on Uncertainty in Artificial Intelligence. pp. 27-34.

Bishop, M., 2006. Pattern Recognition and Machine Learning. Springer.

Blei, D., Lafferty, J., 2006. Correlated topic models. Advances in neural information processing systems 18, 147 .

Blei, D., Lafferty, J., 2009. Topic models. In: Text Mining: Classification, Clustering, and Applications. Chapman \& Hall/CRC Data Mining and Knowledge Discovery Series, pp. 71-89.

Blei, D., Ng, A., Jordan, M., 2003. Latent dirichlet allocation. JMLR 3, 9931022.

Chang, J., Boyd-Graber, J., Gerrish, S., Wang, C., Blei, D., 2009. Reading tea leaves: How humans interpret topic models. In: Advances in Neural Information Processing Systems 22. pp. 288-296.

Cowans, P., 2006. Probabilistic document modelling. Ph.D. thesis, University of Cambridge.

Darling, W., Song, F., 2013. Probabilistic topic and syntax modeling with partof-speech lda. Journal of Computational Linguistics.

Deerwester, S., Dumais, S., Landauer, T., 1990. Indexing by latent semantic analysis. Journal of American Society of Information Science, 391-407.

Ding, Z., Zhang, Q., Huang, X., 2012. Automatic hashtag recommendation for microblogs using topic-specific translation model. In: Proceedings of COLING. pp. 265-274.

Geman, S., Geman, D., 1984. Stochastic relaxation, gibbs distributions, and the bayesian restoration of images. IEEE Transaction on Pattern Analysis and Machine Intelligence 6, 721-741. 
Gilks, W., Richardson, S., Spiegelhalter, D., 1996. Markov Chain Monte Carlo in Practice. Chapman \& Hall, pp. 419-440.

Griffiths, T., Steyvers, M., 2004. Finding scientific topics. In: Proceedings of the National Academy of Sciences. pp. 5228-5235.

Heinrich, G., 2009. Parameter estimation for text analysis. Tech. rep., Fraunhofer IGD.

Minka, T., 2000. Estimating a dirichlet distribution. Tech. rep., MetaCart.

Minka, T., Lafferty, J., 2002. Expectation-propagation for the generative aspect model. In: Proceedings of the 18th Conference on Uncertainty in Artificial Intelligence. pp. 352-359.

Petterson, J., Smola, A., Caetano, T., Buntine, W., Narayanamurthy, S., 2010. Word feature for latent dirichlet allocation. In: Proceedings of Neural Information Processing Systems. pp. 1-9.

Steyvers, M., Griffiths, T., 2007. Probabilistic topic models. Latent Semantic Analysis - A Road to Meaning, 1-15.

Teh, Y., Jordan, M., Beal, M., Blei, D., 2006a. Hierarchical dirichlet processes. Journal of the American Statistical Association 101, 1566-1581.

Teh, Y., Newman, D., M.Welling, 2006b. A collapsed variational bayesian inference algorithm for latent dirichlet allocation. In: Advances in Neural Information Processing Systems 19. pp. 1353-1360.

Thomas, H., 2001. Unsupervised learning by probabilistic latent semantic analysis. Mach Learn 42(1-2), 177-196.

Wallach, H., Mimno, D., McCallum, A., 2009. Rethinking lda: Why priors matter. In: Proceedings of the 23rd Annual Conference on Neural Information Processing Systems. pp. 1973-1981. 
Wang, X., Yao, Y., Ran, C., 2013. Research on a parallel lda modeling method. Beijing University of Technology 33(006), 590-593.

Wikipedia, 2015. http://en.wikipedia.org/wiki/chineselanguage. ph.d. thesis.

Wong, K., Li, W., Xu, R., Zhang, Z., 2009. Introduction to Chinese natural language processing. Morgan \& Claypool.

Xu, T., 2001. Fundamental structural principles of chinese semantic syntax in terms of chinese characters. Applied Linguistics 1, 3-13.

Zhang, Y., Qin, Z., 2010. A topic model of observing chinese characters. In: Proceedings of the 2nd International Conference on Intelligent Human-Machine Systems and Cybernetics (IHMSC). pp. 7-10.

Zhao, Q., Qin, Z., Wan, T., 2011a. Topic modeling of chinese language using character-word relations. In: ICONIP (3). pp. 139-147.

Zhao, Q., Qin, Z., Wan, T., 2011b. What is the basic semantic unit of chinese language? a computational approach based on topic models. In: Meetings on Mathematics of Language (MOL). pp. 143-157. 